\title{
Behavior of OC Curve of Generalized Exponentiated Data
}

\author{
Anwar Hassan ${ }^{1, \star}$, Mehraj Ahmad ${ }^{2}$, Najmus Saquib Hassan ${ }^{3}$ \\ ${ }^{1}$ Department of Statistics, University of Kashmir, Jammu and Kashmir, India \\ ${ }^{2}$ Department of Economics and Statistics, Jammu and Kashmir, India \\ ${ }^{3}$ Agriculture Department of Plant Sciences Shambu Campus, Wollega University Ethiopia
}

\section{ARTICLE INFO}

Article History

Received 09 Mar 2018

Accepted 17 Apr 2020

\section{Keywords}

Operating characteristics curve

Generalized exponential

distribution

Left censored

\section{ABSTRACT}

In this paper a generalized exponential distribution is considered for analyzing left-censored lifetime data as such mechanisms are applicable when the observations become available in an ordered manner with some cases where the origin and the event both occur prior to the start of follow-up. In the present study a test procedure is developed which will approximate a prescribed operating characteristics curve. We also done testing of hypothesis and tried to find values of $\mathrm{r}$ and $\mathrm{C}$ subject to the operating characteristics curve be such that $\mathrm{L}\left(\alpha_{1}\right)=\operatorname{Pr}\left(\right.$ accept $\alpha=\alpha_{1}$ when $\alpha_{1}$ is the true value $)=1-\gamma$ and $\mathrm{L}\left(\alpha_{2}\right)=\operatorname{Pr}\left(\right.$ accept $\alpha=\alpha_{1}$ when $\alpha_{2}$ is the true value $) \leq \beta$. By simulation technique it has been shown that a suitable value of $r$ is to be used for different values of $\gamma$ and $\beta$.

(C) 2020 The Authors. Published by Atlantis Press B.V. This is an open access article distributed under the CC BY-NC 4.0 license (http://creativecommons.org/licenses/by-nc/4.0/).

\section{INTRODUCTION}

Two-parameter generalized exponential (GE) distribution was originally introduced by Gupta and Kundu [1] as a skewed distribution, and as an alternative to Weibull, gamma, or log-normal distribution. Because of the shape and scale parameters, it is observed that GE distribution can take different shapes and it can be used quite effectively to analyze skewed data. Extensive work has been done by several authors on GE distribution. See, e.g., by Gupta and Kundu [2-4], Raqab [5], Raqab and Ahsanullah [6], Zheng [7], and Mitra and Kundu [8] studied the maximum likelihood estimators of the unknown parameters of the GE distribution for left-censored data.

GE distribution, which more accurately represents time to failure, is used instead of more commonly used exponential distribution. Although, incorporation of GE distribution in life testing modeling adds to complexity of modeling and estimation, but due to its flexibility, it fits more accurately to life data than exponential distribution.

The two-parameter GE distribution has the following density function:

$$
\mathrm{f}(\mathrm{x}, \alpha, \lambda)=\alpha \lambda\left(1-\mathrm{e}^{-\lambda \mathrm{x}}\right)^{\alpha-1} \mathrm{e}^{-\lambda \mathrm{x}}, \mathrm{x}>0, \alpha>0, \lambda>0
$$

Cumulative distribution function (cdf)

$$
\mathrm{F}(\mathrm{x}, \alpha, \lambda)=\left(1-\mathrm{e}^{-\lambda \mathrm{x}}\right)^{\alpha}, \mathrm{x}>0, \alpha>0, \lambda>0
$$

Reliability function

$$
\mathrm{R}(\mathrm{x}, \alpha, \lambda)=1-\left(1-\mathrm{e}^{-\lambda \mathrm{x}}\right)^{\alpha}, \mathrm{x}>0, \alpha>0, \lambda>0
$$

Hazard function

$$
h(x, \alpha, \lambda)=\frac{\alpha \lambda\left(1-e^{-\lambda x}\right)^{\alpha-1} e^{-\lambda x}}{1-\left(1-e^{-\lambda x}\right)^{\alpha}}, x>0, \alpha>0, \lambda>0
$$

Here $\alpha>0$ and $\lambda>0$ are the shape and scale parameters respectively. For different values of the shape parameter, the density function can take different shapes. From now on GE distribution with shape parameter $\alpha$ and scale parameter $\lambda$ will be denoted by GE ( $\alpha, \lambda)$. 
There is a widespread application and use of left-censoring or left-censored data in survival analysis and reliability theory, in which a subject is left censored if it is known that the event of interest occurs some time before the recorded follow-up period. For example, a study conducts as investigating factors influencing days to first oestrus in dairy cattle. You start observing your population (for argument's sake) at 40 days after calving but find that several cows in the group have already had an oestrus event. These cows are said to be left censored at day 40 , in medical studies patients are subject to regular examinations. Discovery of a condition only tells us that the onset of sickness fell in the period since the previous examination and nothing about the exact date of the attack. Thus the time elapsed since onset has been left censored. Similarly, we have to handle left-censored data when estimating functions of exact policy duration without knowing the exact date of policy entry; or when estimating functions of exact age without knowing the exact date of birth. A study on the "Patterns of Health Insurance Coverage among Rural and Urban Children" (Coburn et al. [9]) faces this problem due to the incidence of a higher proportion of rural children whose spells were "left censored" in the sample (i.e., those children who entered the sample uninsured), and who remained uninsured throughout the sample. Yet another study (Danzon et al. [10]) which used data on over 900 firms for the period 1988-2000 to estimate the effect on phase-specific (phases 1, 2 and 3) biotech and pharmaceutical R\&D success rates of a firm's overall experience, its experience in the relevant therapeutic category, the diversification of its experience across categories, the industry's experience in the category, and alliances with large and small firms, saw that the data suffered from left censoring. This occurred, e.g., when a phase 2 trial was initiated for a particular indication where there was no information on the phase 1 trial. Application can also be traced in econometric model, e.g., for the joint determination of wages and turnover. Here, after the derivation of the corresponding likelihood function, an appropriate dataset is used for estimation. For a model that is designed for a comprehensive matched employer-employee panel dataset with fairly detailed information on wages, tenure, experience, and a range of other covariates, it may be seen that the raw dataset may contain both completed and uncompleted job spells. A job duration might be incomplete because the beginning of the job spells is not observed, which is an incidence of left censoring (Bagger [11]). For some further examples, one may refer to Balakrishnan and Varadan [12], Lee et al. [13], etc.

Put in general terms, let ...... $\mathrm{X}_{(\mathrm{o})}, \mathrm{X}_{(1)}, \mathrm{X}_{(2)}, \mathrm{X}_{(3)}, \ldots ., \mathrm{X}_{(\mathrm{r})}$ the variable of interest of $\mathrm{n}$ subjects from some GE population is measured, further let first $(n-r)$ subjects are censored at $X_{(1)}$ the $(n-r)^{\text {th }}$ observation and the rest $r$ ordered samples assumed at random from some GE population and the data become available in such a way that the smallest observation comes first, the second smallest second,.........., and finally the largest observation last.

The main aim of this paper is to establishing the Cramer Roa lower bound and efficiency of estimates with respect to Cramer Roa lower bound. Besides that we also derived minimum variance of the biased estimates, also established testing of hypothesis and studied behavior of operating characteristics (OC) curve of GE distribution. From the point of view of acceptance testing, the OC curve based on the r out of $\mathrm{n}$ ordered observations for left-censored data (acceptance region of the form $\hat{\alpha}_{(\mathrm{r}, \mathrm{n})}<\mathrm{C}_{1}$ or $\hat{\alpha}_{(\mathrm{r}, \mathrm{n})}>\mathrm{C}_{2}$ is identical with that based on all $\mathrm{r}$ out of $\mathrm{r}$ observations, details are given in the subsequent sections of the paper.

\section{MAXIMUM LIKELIHOOD ESTIMATION}

In this section, maximum likelihood estimators of the $G E(\alpha, \lambda)$ are derived in presence of left-censored observations. Let $\mathrm{X}_{(1)}, \mathrm{X}_{(2)}, \mathrm{X}_{(3)}, \ldots, \mathrm{X}_{(\mathrm{r})}$ be the last $\mathrm{r}$ order statistics from a random sample of size $n$ following $G E(\alpha, \lambda)$ distribution. For our convenience we denote ordered statistics by $\mathrm{X}_{1}, \mathrm{X}_{2}, \ldots, \mathrm{X}_{\mathrm{r}}$, then the joint probability density function of the ordered statistics is given by

$$
\begin{aligned}
\mathrm{f}\left(\mathrm{x}_{1}, \mathrm{x}_{2}, \ldots, \mathrm{x}_{\mathrm{r}} ; \alpha, \lambda\right) & =\frac{\mathrm{n} !}{\mathrm{r} !}\left(\mathrm{F}\left(\mathrm{x}_{1}\right)\right)^{\mathrm{n}-\mathrm{r}} \mathrm{f}\left(\mathrm{x}_{1}\right) \ldots \mathrm{f}\left(\mathrm{x}_{\mathrm{r}}\right) \\
& =\frac{\mathrm{n} !}{\mathrm{r} !}\left(1-\mathrm{e}^{-\lambda \mathrm{x}_{1}}\right)^{\alpha(\mathrm{n}-\mathrm{r})}(\alpha \lambda)^{\mathrm{r}} \mathrm{e}^{-\lambda \sum_{\mathrm{i}=1}^{\mathrm{r}} \mathrm{x}_{\mathrm{i}}} \prod_{\mathrm{i}=1}^{\mathrm{r}}\left(1-\mathrm{e}^{-\lambda \mathrm{x}_{\mathrm{i}}}\right)^{\alpha-1}
\end{aligned}
$$

The log likelihood function of the observed sample is

$$
\begin{aligned}
\mathrm{L}(\alpha, \lambda)= & \ln (\mathrm{C})+\mathrm{r} \ln (\alpha)+\mathrm{r} \ln (\lambda)-\lambda \sum_{\mathrm{i}=1}^{\mathrm{r}} \mathrm{x}_{\mathrm{i}}+(\alpha-1) \sum_{\mathrm{i}=1}^{\mathrm{r}} \ln \left(1-\mathrm{e}^{-\lambda \mathrm{x}_{\mathrm{i}}}\right) \\
& +\alpha(\mathrm{n}-\mathrm{r}) \ln \left(1-\mathrm{e}^{-\lambda \mathrm{x}_{1}}\right) ; \quad \text { where } \mathrm{C}=\frac{\mathrm{n} !}{\mathrm{r} !}
\end{aligned}
$$

The maximum likelihood estimation (MLE) of $\alpha$ say $\hat{\alpha}_{(\mathrm{r}, \mathrm{n})}$ for known $\lambda$ is

$$
\hat{\alpha}_{(\mathrm{r}, \mathrm{n})}=\hat{\alpha}=-\frac{\mathrm{r}}{\sum_{\mathrm{l}=1}^{\mathrm{r}} \ln \left(1-\mathrm{e}^{-\lambda \mathrm{x}_{\mathrm{i}}}\right)+(\mathrm{n}-\mathrm{r}) \ln \left(1-\mathrm{e}^{-\lambda \mathrm{x}_{1}}\right)}=\frac{\mathrm{r}}{\sum_{\mathrm{l}=1}^{\mathrm{r}} \mathrm{T}_{\mathrm{i}}+(\mathrm{n}-\mathrm{r}) \mathrm{T}_{1}}
$$

$$
\text { where } \mathrm{T}_{\mathrm{i}}=\ln \left(1-\mathrm{e}^{-\lambda \mathrm{x}_{\mathrm{i}}}\right)^{-1} \text { and } \mathrm{T}_{1}=\ln \left(1-\mathrm{e}^{-\lambda \mathrm{x}_{1}}\right)^{-1}
$$


The MLE $\hat{\alpha}_{(\mathrm{r}, \mathrm{n})}$ is the biased estimate; however unbiased estimate can be constructed as

$$
\tilde{\alpha}_{(\mathrm{r}, \mathrm{n})}=\tilde{\alpha}=\frac{\mathrm{r}-1}{\mathrm{r}} \hat{\alpha}_{(\mathrm{r}, \mathrm{n})}=\frac{\mathrm{r}-1}{\sum_{i=1}^{\mathrm{r}} \mathrm{T}_{\mathrm{i}}+(\mathrm{n}-\mathrm{r}) \mathrm{T}_{1}}
$$

Result 1: If $X_{i}$ are random variables independently and identically generalized exponentially distributed GED ( $\left.\alpha, \lambda\right)$, with $\lambda$ known, then $\mathrm{T}_{\mathrm{i}}=\ln \left(1-\mathrm{e}^{-\lambda \mathrm{x}_{\mathrm{i}}}\right)^{-1}$ fallows $\operatorname{Expo}(\alpha)$.

Result 2: $\hat{\alpha}$ the MLE of $\alpha$ has inverted gamma distribution [14] as given below:

$$
\begin{gathered}
\mathrm{f}(\hat{\alpha} \mid \alpha)=\frac{1}{\alpha \Gamma(\mathrm{r}+1)} \mathrm{e}^{\frac{-\mathrm{r} \alpha}{\hat{\alpha}}}\left(\frac{\mathrm{r} \alpha}{\hat{\alpha}}\right)^{\mathrm{r}+1} ; \hat{\alpha} \geq 0, \mathrm{r}, \alpha \geq 0 \\
\mathrm{E}(\hat{\alpha})=\frac{\mathrm{r} \alpha}{\mathrm{r}-1} \text { and } \mathrm{V}(\hat{\alpha})=\frac{\mathrm{r}^{2} \alpha^{2}}{(\mathrm{r}-1)^{2}(\mathrm{r}-2)}
\end{gathered}
$$

Result 3: $\tilde{\alpha}$ the unbiased estimate of $\alpha$ has inverted gamma distribution [14] as given below:

$$
\begin{gathered}
\mathrm{f}(\tilde{\alpha} \mid \alpha)=\frac{1}{\alpha(\mathrm{r}-1) \Gamma \mathrm{r}} \mathrm{e}^{\frac{-(\mathrm{r}-1) \alpha}{\tilde{\alpha}}}\left(\frac{(\mathrm{r}-1) \alpha}{\tilde{\alpha}}\right)^{\mathrm{r}+1} ; \tilde{\alpha} \geq 0, \mathrm{r}>0 \\
\mathrm{E}(\tilde{\alpha})=\alpha \text { and } \mathrm{V}(\tilde{\alpha})=\frac{\alpha^{2}}{(\mathrm{r}-2)}
\end{gathered}
$$

The density (7) depends only on $r$ and not on $n$, this is in fact identical with the density of $\hat{\alpha}_{(r, r)}$, i.e., MLE of $\alpha$ when $r$ out of $r$ observations are tested.

\section{CRAMER RAO LOWER BOUND (CRLB)}

Cramer Rao lower bound unbiased, efficient, and sufficient estimate of MLE and unbiased estimate of the parameter $\alpha$. It is also suggested that an estimate of $\alpha$ which has minimum variance but is biased.

The log likelihood function of the observed sample is

$$
\begin{aligned}
& \mathrm{L}(\alpha, \lambda)=\ln (\mathrm{C})+\mathrm{r} \ln (\alpha)+\mathrm{r} \ln (\lambda)-\lambda \sum_{\mathrm{i}=1}^{\mathrm{r}} \mathrm{x}_{\mathrm{i}}+(\alpha-1) \sum_{\mathrm{i}=1}^{\mathrm{r}} \ln \left(1-\mathrm{e}^{-\lambda \mathrm{x}_{\mathrm{i}}}\right) \\
& +\alpha(\mathrm{n}-\mathrm{r}) \ln \left(1-\mathrm{e}^{-\lambda \mathrm{x}_{1}}\right) ; \quad \text { where } \mathrm{C}=\frac{\mathrm{n} !}{\mathrm{r} !} \\
& \mathrm{L}(\alpha, \lambda)=\ln (\mathrm{C})+\mathrm{r} \ln (\alpha)+\mathrm{r} \ln (\lambda)-\lambda \sum_{\mathrm{i}=1}^{\mathrm{r}} \mathrm{x}_{\mathrm{i}} \\
& \quad+(\alpha-1)\left(\sum_{\mathrm{i}=1}^{\mathrm{r}} \ln \left(1-\mathrm{e}^{-\lambda \mathrm{x}_{\mathrm{i}}}\right)+(\mathrm{n}-\mathrm{r}) \ln \left(1-\mathrm{e}^{-\lambda \mathrm{x}_{1}}\right)\right)+(\mathrm{n}-\mathrm{r}) \ln \left(1-\mathrm{e}^{-\lambda \mathrm{x}_{1}}\right)
\end{aligned}
$$

Using Equation (5) or (6) in Equation (9) we have

$$
\begin{gathered}
=\ln (C)+r \ln (\alpha)+r \ln (\lambda)-\lambda \sum_{i=1}^{r} x_{i}+(\alpha-1)\left(-\frac{r}{\hat{\alpha}}\right)+(n-r) \ln \left(1-e^{-\lambda x_{1}}\right) \\
L(\alpha)=C(\lambda, x)+r \ln (\alpha)-(\alpha-1)\left(\frac{r}{\hat{\alpha}}\right) \\
\frac{\partial L(\alpha)}{\partial \alpha}=\frac{r}{\alpha}-\frac{r}{\hat{\alpha}}
\end{gathered}
$$

Therefore $\mathrm{E}\left(\frac{\partial \mathrm{L}(\alpha)}{\partial \alpha}\right)^{2}=\mathrm{E}\left(\frac{\mathrm{r}}{\alpha}-\frac{\mathrm{r}}{\hat{\alpha}}\right)^{2} \Rightarrow \mathrm{r}^{2} \mathrm{E}\left(\frac{1}{\alpha}-\frac{1}{\hat{\alpha}}\right)^{2}=\mathrm{r}^{2} \mathrm{E}\left(\frac{1}{\alpha^{2}}+\frac{1}{\hat{\alpha}^{2}}-\frac{2}{\alpha \hat{\alpha}}\right)$

$$
\mathrm{E}\left(\frac{\partial \mathrm{L}(\alpha)}{\partial \alpha}\right)^{2}=\mathrm{r}^{2}\left(\frac{1}{\alpha^{2}}+\frac{2}{\alpha^{2}}-\frac{2}{\alpha \alpha}\right) \Rightarrow \mathrm{E}\left(\frac{\partial \mathrm{L}(\alpha)}{\partial \alpha}\right)^{2}=\frac{\mathrm{r}^{2}}{\alpha^{2}}
$$


Therefore Cramer Rao Lower Bound is $\frac{\alpha^{2}}{\mathrm{r}^{2}}$

Efficiency of the estimate $\hat{\alpha}$ with respect MVUE, Efficiency $(\hat{\alpha})=\frac{\alpha^{2}}{\mathrm{r}^{2}} \cdot \frac{(\mathrm{r}-1)^{2}(\mathrm{r}-2)}{(\mathrm{r} \alpha)^{2}}$

$$
\operatorname{Efficiency}(\hat{\alpha})=\left(1-\frac{1}{\mathrm{r}}\right)^{2} \frac{(\mathrm{r}-2)}{\mathrm{r}^{2}}
$$

Efficiency of the estimate $\tilde{\alpha}$ with respect MVUE, Efficiency $(\tilde{\alpha})=\frac{\alpha^{2}}{\mathrm{r}^{2}} \cdot \frac{\mathrm{r}-2}{\alpha^{2}}$

$$
\operatorname{Efficiency}(\tilde{\alpha})=\frac{\mathrm{r}-2}{\mathrm{r}^{2}}
$$

Clearly efficiency of $\hat{\alpha}$ is less than $\tilde{\alpha}$.

Minimum variance biased estimate of $\alpha$ is $\hat{\alpha}_{\mathrm{B}}=\frac{(\mathrm{r}-1) \sqrt{(\mathrm{r}-2)}}{\mathrm{r}^{2}} \hat{\alpha}$ its variance coincides with Cramer Rao Lower Bound (CRLB).

\section{DERIVATION OF TEST BASED ON THE $r$ OUT OF $n$ ORDERED FOLLOW-UP OBSERVATIONS DRAWN FROM GED}

In this section we develop a best test on the first $\mathrm{r}$ ordered observations (from a sample of size $\mathrm{n}$ ) so as to decide between two values of $\alpha, \alpha_{1} \& \alpha_{2}$ i.e. $H_{0}: \alpha=\alpha_{1}$ and $H_{0}: \alpha=\alpha_{2}$. Case I: when $\alpha_{1}>\alpha_{2}$ and case II: when $\alpha_{1}<\alpha_{2}$.

By the best test we mean according to the usual Neyman-Pearson terminology a test which has the property that among all tests having a fixed probability $\gamma$ size of rejecting $\alpha=\alpha_{1}$ when it is true, the test in question will have the largest possible chance of rejecting $\alpha=\alpha_{1}$ when the alternative $\alpha=\alpha_{2}$ is true.

To derive the best test we use Neyman-Pearson (NP) lemma, according to the lemma a best test must be one for which the region of rejection can be found from the inequality.

$$
\begin{gathered}
\frac{\mathrm{L}\left(\mathrm{x}_{1}, \mathrm{x}_{2}, \ldots, \mathrm{x}_{\mathrm{r}}, \alpha_{2}\right)}{\mathrm{L}\left(\mathrm{x}_{1}, \mathrm{x}_{2}, \ldots, \mathrm{x}_{\mathrm{r}}, \alpha_{1}\right)}>\mathrm{k} \\
\frac{\mathrm{n} !}{\mathrm{r} !} \alpha_{2}^{\mathrm{r}} \lambda^{\mathrm{r}} \prod_{\mathrm{i}=1}^{\mathrm{r}}\left(1-\mathrm{e}^{-\lambda \mathrm{x}_{\mathrm{i}}}\right)^{\alpha_{2}-1} \mathrm{e}^{-\lambda \sum_{\mathrm{i}=1}^{\mathrm{r}} \mathrm{x}_{\mathrm{i}}}\left(1-\mathrm{e}^{-\lambda \mathrm{x}_{1}}\right)^{\alpha_{2}(\mathrm{n}-\mathrm{r})} \\
\frac{\mathrm{n} !}{\mathrm{r} !} \alpha_{1}^{\mathrm{r}} \lambda \mathrm{r} \prod_{\mathrm{i}=1}^{\mathrm{r}}\left(1-\mathrm{e}^{-\lambda \mathrm{x}_{\mathrm{i}}}\right)^{\alpha_{1}-1} \mathrm{e}^{-\lambda \sum_{\mathrm{i}=1}^{\mathrm{r}} \mathrm{x}_{\mathrm{i}}}\left(1-\mathrm{e}^{-\lambda \mathrm{x}_{1}}\right)^{\alpha_{1}(\mathrm{n}-\mathrm{r})} \\
\left(\alpha_{2}-\alpha_{1}\right)\left[\sum_{\mathrm{i}=1}^{\mathrm{r}} \ln \left(1-\mathrm{e}^{-\lambda \mathrm{x}_{\mathrm{i}}}\right)+(\mathrm{n}-\mathrm{r}) \ln \left(1-\mathrm{e}^{-\lambda \mathrm{x}_{1}}\right)\right]>\ln \left(\mathrm{k}_{1}\right)
\end{gathered}
$$

where $\mathrm{k}_{1}=\left(\mathrm{k}\left(\frac{\alpha_{1}}{\alpha_{2}}\right)^{\mathrm{r}}\right)$

Case I: when $\alpha_{1}>\alpha_{2}$

Because $\alpha_{1}$ and $\alpha_{2}$ are preassigned constants such that $\alpha_{1}>\alpha_{2}$, and using Equation (5) we have

$$
\left(\alpha_{2}-\alpha_{1}\right)\left[-\frac{\mathrm{r}}{\hat{\alpha}}\right]>\ln \left(\mathrm{k}_{1}\right) \Rightarrow \frac{\mathrm{r}\left(\alpha_{1}-\alpha_{2}\right)}{\hat{\alpha}}>\ln \left(\mathrm{k}_{1}\right)
$$

Since $\alpha_{1}$ and $\alpha_{2}$ are preassigned constants such that $\alpha_{1}>\alpha_{2}$. It fallows at once that the region of rejection/critical region for $\alpha=\alpha_{1}$ is

$$
\hat{\alpha}<\frac{\mathrm{r}\left(\alpha_{1}-\alpha_{2}\right)}{\ln \left(\mathrm{k}_{1}\right)} \Rightarrow \hat{\alpha}<\mathrm{C}_{1}, \quad \text { where } \mathrm{C}_{1}=\frac{\mathrm{r}\left(\alpha_{1}-\alpha_{2}\right)}{\ln \left(\mathrm{k}_{1}\right)}
$$

i.e., best critical region is

$$
\mathrm{W}_{1}=\left\{\mathrm{X}: \hat{\alpha}<\mathrm{C}_{1}\right\}
$$

Case II when $\alpha_{1}<\alpha_{2}$ :

$$
\left(\alpha_{2}-\alpha_{1}\right)\left[-\frac{\mathrm{r}}{\hat{\alpha}}\right]>\ln \left(\mathrm{k}_{2}\right) \Rightarrow \frac{-\mathrm{r}\left(\alpha_{2}-\alpha_{1}\right)}{\hat{\alpha}}>\ln \left(\mathrm{k}_{2}\right) ; \quad \text { where } \mathrm{k}_{2}=\left(\mathrm{k}\left(\frac{\alpha_{2}}{\alpha_{1}}\right)^{-\mathrm{r}}\right)
$$


Since $\alpha_{1}$ and $\alpha_{2}$ are preassigned constants such that $\alpha_{2}>\alpha_{1}$. It fallows at once that the region of rejection/critical region for $\alpha=\alpha_{1}$ is

$$
\hat{\alpha}>-\frac{\mathrm{r}\left(\alpha_{2}-\alpha_{1}\right)}{\ln \left(\mathrm{k}_{2}\right)} \Rightarrow \hat{\alpha}>\mathrm{C}_{2}, \quad \text { where } \mathrm{C}_{2}=-\frac{\mathrm{r}\left(\alpha_{2}-\alpha_{1}\right)}{\ln \left(\mathrm{k}_{2}\right)}
$$

i.e., best critical region is

$$
\mathrm{W}_{2}=\left\{\mathrm{X}: \hat{\alpha}>\mathrm{C}_{2}\right\}
$$

\section{DETERMINATION OF CONSTANTS $C_{1}$ AND $C_{2}$}

The constants $\mathrm{C}_{1}$ and $\mathrm{C}_{2}$ are so chosen as to make the probability of each of the relations (10) and (11) equal to $\gamma$ when the null hypothesis $\mathrm{H}_{\mathrm{o}}$ is true or in other words to meet the condition that the probability of rejecting $\alpha=\alpha_{1}$ when true value equals $\gamma$, we need to choose $\mathrm{C}_{1}$ and $\mathrm{C}_{2}$ so that

Case I when $\alpha_{1}>\alpha_{2}$ :

$$
\mathrm{P}\left\{\hat{\alpha}>\mathrm{C}_{1} \mid \alpha=\alpha_{1}\right\}=\gamma \Rightarrow \mathrm{P}\left\{\frac{1}{\hat{\alpha}}>\frac{1}{\mathrm{C}_{1}} \mid \mathrm{H}_{\mathrm{o}}\right\}=\gamma
$$

To find $\mathrm{C}_{1}$ explicitly we use the result which states that $\hat{\alpha}$ has $(7)$ as its probability density function and it can be very easily verified that $\frac{\mathrm{r} \alpha}{\hat{\alpha}} \sim \operatorname{Gamma}(\mathrm{r})$

Thus $\frac{2 \mathrm{r} \alpha}{\hat{\alpha}} \sim \operatorname{Gamma}\left(\frac{1}{2}, \mathrm{r}\right) \mathrm{i}, \mathrm{e} \chi_{2 \mathrm{r}}^{2}$ is a random variable which is distributed as chi-square with $2 \mathrm{r}$ degrees of freedom. Thus above probability relation can be written as

$$
\mathrm{P}\left\{\frac{2 \mathrm{r} \alpha}{\hat{\alpha}}>\left.\frac{2 \mathrm{r} \alpha_{1}}{\mathrm{C}_{1}}\right|_{\alpha=\alpha_{1}}\right\}=\gamma \Rightarrow \mathrm{P}\left\{\chi^{2}(2 \mathrm{r})>\left.\frac{2 \mathrm{r} \alpha_{1}}{\mathrm{C}_{1}}\right|_{\alpha=\alpha_{1}}\right\}=\gamma
$$

Let us denote a chi-square variable with $\mathrm{n}$ degrees of freedom as $\chi^{2}(\mathrm{n})$ and let us define the constant $\chi_{\gamma}^{2}(\mathrm{n})$ by the equality $\mathrm{P}\left[\chi^{2}(\mathrm{n})>\chi_{\gamma}^{2}(\mathrm{n})\right]=\gamma$, where $\chi_{\gamma}^{2}(\mathrm{n})$ is the upper $100 \gamma$ per cent point.

Then (12) can be written as $\frac{2 \mathrm{r} \alpha_{1}}{\mathrm{C}_{1}}=\chi_{\gamma}^{2}(2 \mathrm{r})$, this gives $\mathrm{C}_{1}=\frac{2 \mathrm{r} \alpha_{1}}{\chi_{\gamma}^{2}(2 \mathrm{r})}$.

Hence (12) will be satisfied if the region of rejection for $\alpha=\alpha_{1}$ is given by

$$
\mathrm{W}_{1}=\left\{\mathrm{X}: \hat{\alpha}<\frac{2 \mathrm{r} \alpha_{1}}{\chi_{\gamma}^{2}(2 \mathrm{r})}\right\}
$$

It is convenient in what follows to use acceptance rather than rejection regions. Consequently the NP theory tells us that a simple test for $\alpha=\alpha_{1}$ against $\alpha<\alpha_{1}$ with type-I error equal to $\gamma$ is given by an acceptance region of the form

$$
\mathrm{A}_{1}=\left\{\mathrm{X}: \hat{\alpha}>\frac{2 \mathrm{r} \alpha_{1}}{\chi_{\gamma}^{2}(2 \mathrm{r})}\right\}
$$

Case II when $\alpha_{1}<\alpha_{2}$ : Now constant $C_{2}$ can be obtained as $\mathrm{P}\left\{\hat{\alpha}>\mathrm{C}_{2} \mid \alpha=\alpha_{1}\right\}=\gamma$

$$
\begin{gathered}
\mathrm{P}\left\{\frac{1}{\hat{\alpha}}<\frac{1}{\mathrm{C}_{2}} \mid \mathrm{H}_{\mathrm{o}}\right\}=\gamma \Rightarrow \mathrm{P}\left\{\frac{2 \mathrm{r} \alpha}{\hat{\alpha}}<\left.\frac{2 \mathrm{r} \alpha_{1}}{\mathrm{C}_{2}}\right|_{\alpha=\alpha_{1}}\right\}=\gamma \\
\mathrm{P}\left\{\chi^{2}(2 \mathrm{r})>\left.\frac{2 \mathrm{r} \alpha_{1}}{\mathrm{C}_{2}}\right|_{\alpha=\alpha_{1}}\right\}=1-\gamma
\end{gathered}
$$

Thus in a analogue as discussed earlier, the above probability equation can yield $\frac{2 \mathrm{r} \alpha_{1}}{\mathrm{C}_{2}}=\chi_{1-\gamma}^{2}(2 \mathrm{r})$, this gives $\mathrm{C}_{2}=\frac{2 \mathrm{r} \alpha_{1}}{\chi_{1-\gamma}^{2}(2 \mathrm{r})}$.

The best critical region can be written in the form as given below

$$
\mathrm{W}_{2}=\left\{\mathrm{X}: \hat{\alpha}>\frac{2 \mathrm{r} \alpha_{1}}{\chi_{1-\gamma}^{2}(2 \mathrm{r})}\right\}
$$

It is convenient in what fallows to use acceptance rather than rejection regions. Consequently the NP theory tells us that a simple test for $\alpha=\alpha_{1}$ against $\alpha>\alpha_{1}$ with type-I error equal to $\gamma$ is given by an acceptance region of the form

$$
\mathrm{A}_{2}=\left\{\mathrm{X}: \hat{\alpha}<\frac{2 \mathrm{r} \alpha_{1}}{\chi_{1-\gamma}^{2}(2 \mathrm{r})}\right\}
$$




\section{POWER FUNCTION OF THE TEST}

Case I when $\alpha_{1}>\alpha_{2}$ : The power function of the test is

$$
\begin{gathered}
\text { Power }=1-\beta=P\left\{\left.X \in \mathrm{W}_{1}\right|_{\mathrm{H}_{1}}\right\}=\mathrm{P}\left\{\chi^{2}(2 \mathrm{r})>\left.\frac{2 \mathrm{r} \alpha_{2}}{\mathrm{C}_{1}}\right|_{\alpha=\alpha_{2}}\right\} \\
\Rightarrow \text { Power }=\mathrm{P}\left\{\chi^{2}(2 \mathrm{r})>\frac{\alpha_{2}}{\alpha_{1}} \chi_{\gamma}^{2}(2 \mathrm{r})\right\}
\end{gathered}
$$

According to NP lemma the region of rejection (13) has a greater chance of rejecting $\alpha=\alpha_{1}$ when $\alpha=\alpha_{2}$ is true than any other region which assigns probability $\gamma$ to the rejection of $\alpha=\alpha_{1}$ when $\alpha_{1}$ is the true value. Evidently the region (13) does not depend on the particular choice of alternative $\alpha$. Therefore the region (13) gives a uniformly most powerful test in the NP sense of the hypothesis $\alpha=\alpha_{1}$ against $\alpha<\alpha_{1}$.

Case II when $\alpha_{1}<\alpha_{2}$ : The power function of the test is

$$
\begin{gathered}
\text { Power }=1-\beta=P\left\{\left.X \in \mathrm{W}_{2}\right|_{\mathrm{H}_{1}}\right\}=\mathrm{P}\left\{\chi^{2}(2 \mathrm{r})<\left.\frac{2 \mathrm{r} \alpha_{2}}{\mathrm{C}_{2}}\right|_{\alpha=\alpha_{2}}\right\} \\
\Rightarrow \text { Power }=\mathrm{P}\left\{\chi^{2}(2 \mathrm{r})<\frac{\alpha_{2}}{\alpha_{1}} \chi_{1-\gamma}^{2}(2 \mathrm{r})\right\}
\end{gathered}
$$

According to NP lemma the region of rejection (15) has a greater chance of rejecting $\alpha=\alpha_{1}$ when $\alpha=\alpha_{2}$ is true than any other region which assigns probability $\gamma$ to the rejection of $\alpha=\alpha_{1}$ when $\alpha_{1}$ is the true value. Evidently the region (15) does not depend on the particular choice of alternative $\alpha$. Therefore the region (15) gives a uniformly most powerful test in the NP sense of the hypothesis $\alpha=\alpha_{1}$ against $\alpha>\alpha_{1}$.

\section{OC FUNCTION}

Case I when $\alpha_{1}>\alpha_{2}$ : Let us now look at the OC curve of a procedure specified by (14), i.e., let us study

$$
\begin{gathered}
\mathrm{L}(\alpha)=\text { Probability of accepting } \alpha=\alpha_{1} \text { when } \alpha \text { is the true value } \\
\mathrm{L}(\alpha)=\mathrm{P}\left\{\hat{\alpha}>\frac{2 \mathrm{r} \alpha_{1}}{\chi_{\gamma}^{2}(2 \mathrm{r})}\right\} \\
\mathrm{L}(\alpha)=1-\mathrm{P}\left\{\chi^{2}(2 \mathrm{r})>\frac{\alpha}{\alpha_{1}} \chi_{\gamma}^{2}(2 \mathrm{r})\right\}
\end{gathered}
$$

The graph of $L(\alpha)$ for various values of $r$ and of the ratio $\frac{\alpha}{\alpha_{1}}$ when $\gamma=0.05$ is given in Figure 1 .

In the problem just discussed, it was assumed that $\mathrm{r}$ and $\gamma$ are known and $\mathrm{C}_{1}$ is unknown. We shall now consider a problem where both $\mathrm{r}$ and $\mathrm{C}_{1}$ are initially unknown. We want to choose these unknowns in such a way that the resulting OC curve will have the property that

$$
\mathrm{L}\left(\alpha_{1}\right)=1-\gamma \text { and } \mathrm{L}\left(\alpha_{2}\right) \leq \beta
$$

where $\alpha_{2} \leq \alpha_{1}$ and $\gamma$ and $\beta$ are prescribed in advance. To meet conditions (18) means substituting $\alpha_{2}$ for $\alpha$ in (17) and requiring that $r$ be such that

$$
\begin{gathered}
\mathrm{L}\left(\alpha_{2}\right) \leq \beta \Rightarrow \mathrm{P}\left\{\chi^{2}(2 \mathrm{r})<\frac{\alpha_{2}}{\alpha_{1}} \chi_{\gamma}^{2}(2 \mathrm{r})\right\} \leq \beta \\
\text { or } \mathrm{P}\left\{\chi^{2}(2 \mathrm{r})>\frac{\alpha_{2}}{\alpha_{1}} \chi_{\gamma}^{2}(2 \mathrm{r})\right\} \geq 1-\beta
\end{gathered}
$$

This implies that

$$
\frac{\alpha_{2}}{\alpha_{1}} \chi_{\gamma}^{2}(2 \mathrm{r}) \leq \chi_{1-\beta}^{2}(2 \mathrm{r}) \Rightarrow \frac{\alpha_{1}}{\alpha_{2}} \geq \frac{\chi_{\gamma}^{2}(2 \mathrm{r})}{\chi_{1-\beta}^{2}(2 \mathrm{r})}
$$




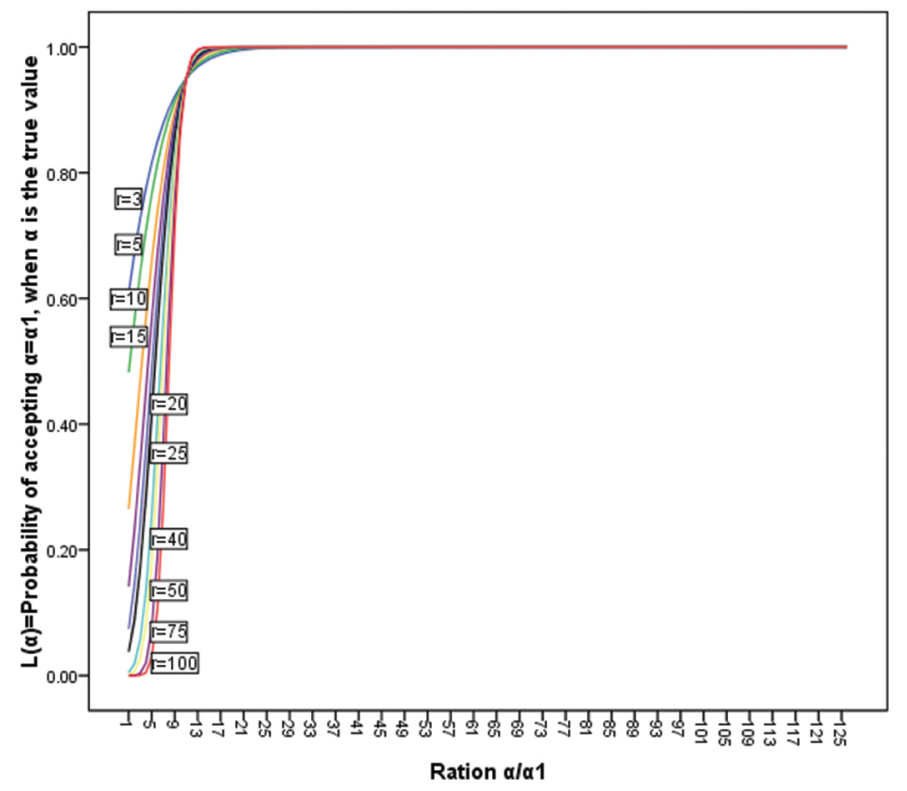

Figure $1 \mid \mathrm{OC}$ of fests of the form $\operatorname{MLE}(\alpha)>\mathrm{C} 1, \mathrm{~L}(\alpha)=0.95$

Knowing (19) makes it an easy matter to find that integer $\mathrm{r}$ which ensures that the OC curve pass most nearly through the points $\left[\alpha_{1}, L\left(\alpha_{1}\right)=1-\gamma\right]$ and $\left[\alpha_{2}, L\left(\alpha_{2}\right)=\beta\right]$. It can be verified that as $r$ goes through the values $1,2,3, \ldots \ldots$ the ratio $\chi_{\gamma}^{2}(2 \mathrm{r}) / \chi_{1-\beta}^{2}(2 \mathrm{r})$ is strictly decreasing and it is easy to show that it tends to zero. Consequently there is a smaller integer $r$ such that $\frac{\alpha_{1}}{\alpha_{2}} \geq \frac{\chi_{\gamma}^{2}(2 r)}{\chi_{1-\beta}^{2}(2 r)}$

This is the value of $r$ which we wish to use. If with this value of $r$ we use an acceptance region $\alpha=\alpha_{1}$ of the form $\hat{\alpha}>C_{1}$ where $C_{1}=\frac{2 r \alpha_{1}}{\chi_{\gamma}^{2}(2 r)}$ We shall have a test whose OC curve is such that $\mathrm{L}\left(\alpha_{1}\right)=1-\gamma$ and $\mathrm{L}\left(\alpha_{2}\right) \leq \beta$. Incidentally, a region of acceptance for $\alpha=\alpha_{1}$ of the form $\hat{\alpha}>C_{1}^{*}$ where $C_{1}^{*}=\frac{2 \mathrm{r} \alpha_{2}}{\chi_{1-\beta}^{2}(2 \mathrm{r})}$ will give for same $\mathrm{r}$ an OC curve such that $\mathrm{L}\left(\alpha_{1}\right) \leq 1-\gamma$ and $\mathrm{L}\left(\alpha_{2}\right)=\beta$.

Case II when $\alpha_{1}<\alpha_{2}$ : Let us now look at the OC curve of a procedure specified by (16), i.e., let us study

$\mathrm{L}(\alpha)=$ Probability of accepting $\alpha=\alpha_{1}$ when $\alpha$ is the true value

$$
\begin{gathered}
\mathrm{L}(\alpha)=\mathrm{P}\left\{\hat{\alpha}<\frac{2 \mathrm{r} \alpha_{1}}{\chi_{1-\gamma}^{2}(2 \mathrm{r})}\right\} \Rightarrow \mathrm{L}(\alpha)=\mathrm{P}\left\{\frac{2 \mathrm{r} \alpha}{\hat{\alpha}}>\frac{\alpha \chi_{1-\gamma}^{2}(2 \mathrm{r})}{\alpha_{1}}\right\} \\
\mathrm{L}(\alpha)=\mathrm{P}\left\{\chi^{2}(2 \mathrm{r})>\frac{\alpha}{\alpha_{1}} \chi_{1-\gamma}^{2}(2 \mathrm{r})\right\}
\end{gathered}
$$

The graph of $L(\alpha)$ for various values of $r$ and of the ratio $\frac{\alpha}{\alpha_{1}}$ when $\gamma=0.05$ is given in Figure 2 .

In the problem just discussed, it was assumed that $r$ and $\gamma$ are known and $C_{2}$ is unknown. We shall now consider a problem where both $r$ and $\mathrm{C}_{2}$ are initially unknown. We want to choose these unknowns in such a way that the resulting $\mathrm{OC}$ curve will have the property that

$$
\mathrm{L}\left(\alpha_{1}\right)=1-\gamma \text { and } \mathrm{L}\left(\alpha_{2}\right) \leq \beta
$$

where $\alpha_{2} \geq \alpha_{1}$ and $\gamma$ and $\beta$ are prescribed in advance. To meet conditions (21) means substituting $\alpha_{2}$ for $\alpha$ in (20) and requiring that $r$ be such that

$$
\mathrm{L}\left(\alpha_{2}\right) \leq \beta
$$

$$
\mathrm{P}\left\{\chi^{2}(2 \mathrm{r})>\frac{\alpha_{2}}{\alpha_{1}} \chi_{1-\gamma}^{2}(2 \mathrm{r})\right\} \leq \beta
$$




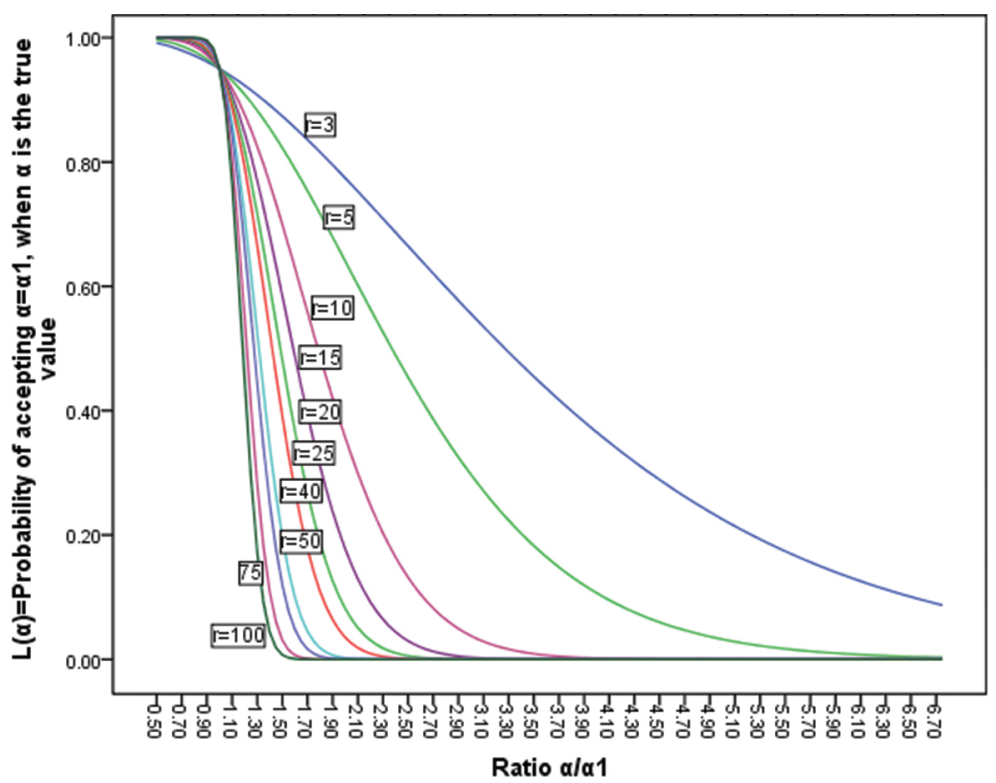

Figure 2 Operating characterstics of tests of the form $\operatorname{MLE}(\alpha)<\mathrm{C} 2, \mathrm{~L}(\alpha 1)=0.95$

This implies that

$$
\frac{\alpha_{2}}{\alpha_{1}} \chi_{1-\gamma}^{2}(2 \mathrm{r}) \geq \chi_{\beta}^{2}(2 \mathrm{r}) \Rightarrow \frac{\alpha_{2}}{\alpha_{1}} \geq \frac{\chi_{\beta}^{2}(2 \mathrm{r})}{\chi_{1-\gamma}^{2}(2 \mathrm{r})}
$$

Knowing (22) makes it an easy matter to find that integer $\mathrm{r}$ which ensures that the OC curve pass most nearly through the points $\left[\alpha_{1}, \mathrm{~L}\left(\alpha_{1}\right)=1-\gamma\right]$ and $\left[\alpha_{2}, \mathrm{~L}\left(\alpha_{2}\right)=\beta\right]$. It can be verified that as $\mathrm{r}$ goes through the values $1,2,3, \ldots \ldots$ the ratio $\chi_{\beta}^{2}(2 \mathrm{r}) / \chi_{1-\gamma}^{2}(2 \mathrm{r})$ is strictly decreasing and it is easy to show that it tends to zero. Consequently there is a smaller integer $\mathrm{r}$ such that $\frac{\alpha_{2}}{\alpha_{1}} \geq \frac{\chi_{\beta}^{2}(2 \mathrm{r})}{\chi_{1-\gamma}^{2}(2 \mathrm{r})}$

This is the value of $r$ which we wish to use. If with this value of $r$ we use an acceptance region $\alpha=\alpha_{1}$ of the form $\hat{\alpha}<\mathrm{C}_{2}$ where $\mathrm{C}_{2}=\frac{2 \mathrm{r} \alpha_{1}}{\chi_{1-\gamma}^{2}(2 \mathrm{r})}$. We shall have a test whose OC curve is such that $\mathrm{L}\left(\alpha_{1}\right)=1-\gamma$ and $\mathrm{L}\left(\alpha_{2}\right) \leq \beta$. Incidentally, a region of acceptance for $\alpha=\alpha_{1}$ of the form $\hat{\alpha}>C_{2}^{*}$ where $C_{2}^{*}=\frac{2 r \alpha_{2}}{\chi_{\beta}^{2}(2 r)}$ will give for same $r$ an OC curve such that $L\left(\alpha_{1}\right) \leq 1-\gamma$ and $L\left(\alpha_{2}\right)=\beta$.

\section{UNIFORMLY MOST POWERFUL CRITICAL REGION}

Case I: when $\alpha_{1}>\alpha_{2}$

The best critical region as given in (13) is $\mathrm{W}_{1}=\left\{\mathrm{X}: \hat{\alpha}<\frac{2 \mathrm{r} \alpha_{1}}{\chi_{\gamma}^{2}(2 \mathrm{r})}\right\}$

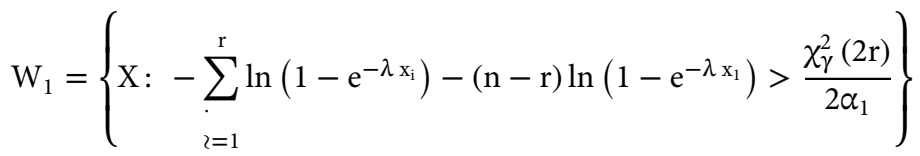

It is independent of $\alpha_{2}$, i.e., alternative value of $\alpha$, therefore $W_{1}$ is uniformly most powerful critical region for testing $H_{0}: \alpha=\alpha \alpha_{1}$ against $\mathrm{H}_{1}: \alpha=\alpha_{2}<\alpha_{1}$, this implies that no choice of $\alpha_{2}$ can change the size of critical region for $\alpha_{2}<\alpha_{1}$. 
Case II: when $\alpha_{2}>\alpha_{1}$

The best critical region as given in $(15)$ is $\mathrm{W}_{2}=\left\{\mathrm{X}: \hat{\alpha}>\frac{2 \mathrm{r} \alpha_{1}}{\chi_{1-\gamma}^{2}(2 \mathrm{r})}\right\}$

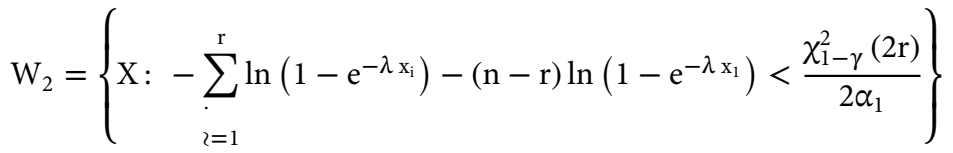

It is independent of $\alpha_{2}$, i.e., alternative value of $\alpha$, therefore $\mathrm{W}_{2}$ is also uniformly most powerful critical region for testing $\mathrm{H}_{\mathrm{o}}: \alpha=\alpha_{1}$ against $\mathrm{H}_{1}: \alpha=\alpha_{2}>\alpha_{1}$, this implies that no choice of $\alpha_{2}$ can change the size of critical region for $\alpha_{2}>\alpha_{1}$.

However, since the two critical regions $\mathrm{W}_{1}$ and $\mathrm{W}_{2}$ are different, i.e., $\mathrm{W}_{1} \cap \mathrm{W}_{2}=\varnothing$, therefore there exists no critical region of size $\gamma$ which is uniformly most powerful for testing $\mathrm{H}_{\mathrm{o}}: \alpha=\alpha_{1}$ against the two-tailed alternative $\mathrm{H}_{1}: \alpha \neq \alpha_{1}$.

If $\frac{\alpha_{1}}{\alpha_{2}}=3$ and $\gamma=\beta=0.01$, it is easy to show that a suitable $\mathrm{r}$ to use is 18 . If $\frac{\alpha_{1}}{\alpha_{2}}=3$ and $\gamma=0.05, \beta=0.01$, then the proper $\mathrm{r}$ is 14 . Similarly If $\frac{\alpha_{1}}{\alpha_{2}}=3$ and $\gamma=0.1, \beta=0.01$ then proper $\mathrm{r}$ is 12 . This means, for instance that if we want the test procedure to accept a lot whose life characteristics is If $\alpha_{2}=500$ hours only $5 \%$ of the times, then it is possible to draw valid inference while observing only 9 observations $\mathrm{x}_{1 \mathrm{n}}, \mathrm{x}_{2 \mathrm{n}}, \ldots ., \mathrm{x}_{9 \mathrm{n}}$ although rest (n-9) are left censored, and if $\hat{\alpha}>935$ hours accept $\alpha=\alpha_{1}$, if $\hat{\alpha}<935$ hours accept $\alpha=\alpha_{2}$. Such a procedure will have an OC curve for which $\mathrm{L}\left(\alpha_{1}\right)=0.95$ and $\mathrm{L}\left(\alpha_{2}\right) \leq 0.05$. It should be noted that " $\mathrm{n}$ " number of items tested is left arbitrary. If one's object is to reduce testing time, then it is clearly advisable from Table 1 to make " $n$ " more than 9.

Table 1 Values of $\mathrm{r}$ and acceptance regions for fixed $\gamma, \beta$ where $\alpha=$ probability of rejecting $\alpha_{1}$ when $\alpha=\alpha_{1} ; \beta=$ probability of accepting $\alpha_{1}$ when $\alpha=\alpha_{1}$; i.e., $\beta=$ probability of accepting $\alpha_{1}$ when $\alpha=\alpha_{2}$; for both cases $\alpha_{1}>\alpha_{2}$ and $\alpha_{1}<\alpha_{2}$. Acceptance regions are of the form $\hat{\alpha}>\mathrm{C}_{1}$, $\hat{\alpha}<\mathrm{C}_{2}$.

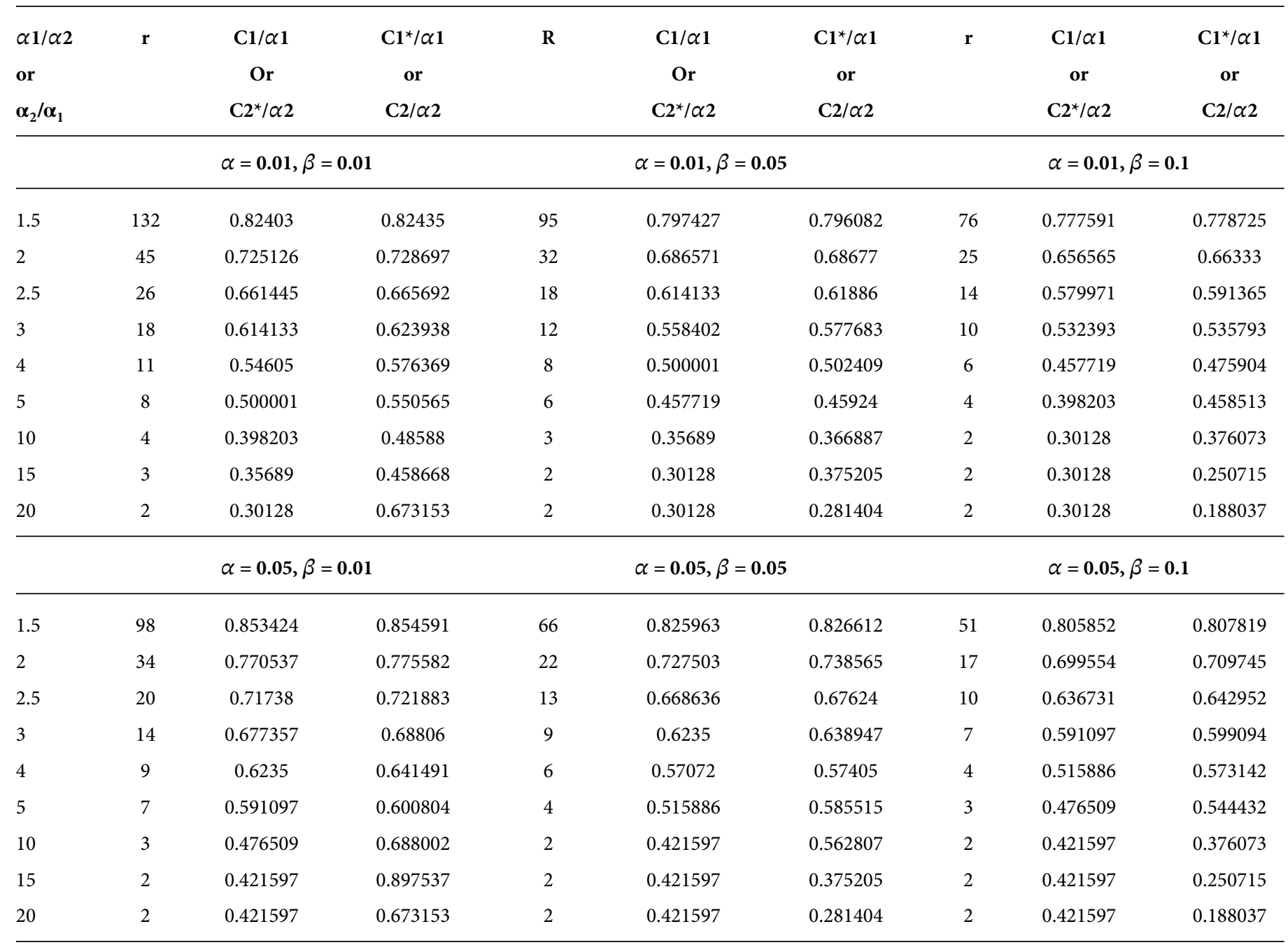


Table 1 Values of $\mathrm{r}$ and acceptance regions for fixed $\gamma, \beta$ where $\alpha=$ probability of rejecting $\alpha_{1}$ when $\alpha=\alpha_{1}$; $\beta=$ probability of accepting $\alpha_{1}$ when $\alpha=\alpha_{1}$; i.e., $\beta=$ probability of accepting $\alpha_{1}$ when $\alpha=\alpha_{2}$; for both cases $\alpha_{1}>\alpha_{2}$ and $\alpha_{1}<\alpha_{2}$. Acceptance regions are of the form $\hat{\alpha}>\mathrm{C}_{1}$, $\hat{\alpha}<\mathrm{C}_{2}$. (Continued)

\begin{tabular}{|c|c|c|c|c|c|c|c|c|c|}
\hline \multirow[b]{2}{*}{1.5} & \multicolumn{3}{|c|}{$\alpha=0.1, \beta=0.01$} & \multicolumn{3}{|c|}{$\alpha=0.1, \beta=0.05$} & \multicolumn{3}{|c|}{$\alpha=0.1, \beta=0.1$} \\
\hline & 82 & 0.87422 & 0.875869 & 53 & 0.84776 & 0.539311 & 40 & 0.828344 & 0.829731 \\
\hline 2 & 29 & 0.803771 & 0.807497 & 18 & 0.762515 & 0.352952 & 14 & 0.738476 & 0.739206 \\
\hline 2.5 & 17 & 0.757185 & 0.764511 & 10 & 0.703928 & 0.254692 & 8 & 0.679641 & 0.687268 \\
\hline 3 & 12 & 0.722973 & 0.736895 & 7 & 0.664637 & 0.197032 & 5 & 0.625501 & 0.685141 \\
\hline 4 & 8 & 0.679641 & 0.688206 & 5 & 0.625501 & 0.13656 & 3 & 0.563664 & 0.68054 \\
\hline 5 & 6 & 0.646923 & 0.672162 & 3 & 0.563664 & 0.095302 & 2 & 0.514176 & 0.752146 \\
\hline 10 & 3 & 0.563664 & 0.688002 & 2 & 0.514176 & 0.04216 & 2 & 0.514176 & 0.376073 \\
\hline 15 & 2 & 0.514176 & 0.897537 & 2 & 0.514176 & 0.028106 & 2 & 0.514176 & 0.250715 \\
\hline 20 & 2 & 0.514176 & 0.673153 & 2 & 0.514176 & 0.02108 & 2 & 0.514176 & 0.188037 \\
\hline
\end{tabular}

\section{ACKNOWLEDGEMENTS}

The authors appreciate and thank the referee and the editor for many helpful comments and suggestions, which substantially simplified the paper.

\section{REFERENCES}

1. R.D. Gupta, D. Kundu, Aust. N. Z. J. Stat. (1999), 41 173-188.

2. R.D. Gupta, D. Kundu, J. Stat. Theory Appl. 1 (2002), 101-118.

3. R.D. Gupta, D. Kundu, J. Stat. Comput. Simul. 69 (2001), 315-338.

4. R.D. Gupta, D. Kundu, Biom. J. 43 (2001), 117-130.

5. M.Z. Raqab, J. Stat. Plan. Inference. 104 (2002), 339-350.

6. M.Z. Raqab, M. Ahsanullah, J. Stat. Comput. Simul. 69 (2001), 109-124.

7. G. Zheng, Biom. J. 44 (2002), 353-357.

8. S. Mitra, D. Kundu, J. Stat. Comput. Simul. 78 (2008), 669-679.

9. F. Coburn, T. McBride, E. Ziller, Patterns of Health Insurance Coverage among Rural and Urban Children, Working Paper No. 26, Maine Rural Health Research Center, Edmund S. Muskie School of Public Service, University of Southern Maine, Portland, ME, USA, 2001.

10. P.M. Danzon, S. Nicholson, N.S. Pereira, Productivity in Pharmaceutical- Biotechnology R\&D: The Role of Experience and Alliances, University of Pennsylvania, The Wharton School, 2004.

11. J. Bagger, Wage Growth and Turnover in Denmark, University of Aarhus, Denmark, 2005.

12. N. Balakrishnan, J. Varadan, IEEE Trans. Reliab. 40 (1991), 146-151.

13. K.R. Lee, C.H. Kapadia, B.B. Dwight, Stat. Hefte. 21 (1980), 14-21.

14. H. Raiffa, R. Schlaifer, Applied Statistical Decision Theory, Harvard University Press, 1961. 